\title{
Electron tunneling through double magnetic barriers on the surface of a topological insulator
}

\author{
Zhenhua Wu, ${ }^{1}$ F. M. Peeters, ${ }^{2}$ and Kai Chang ${ }^{1}$ \\ ${ }^{1}$ SKLSM, Institute of Semiconductors, Chinese Academy of Sciences, P.O. Box 912, 100083 Beijing, China \\ ${ }^{2}$ Department of Physics, University of Antwerp, Groenenborgerlaan 171, B-2020 Antwerpen, Belgium \\ (Received 24 April 2010; revised manuscript received 24 August 2010; published 24 September 2010)
}

\begin{abstract}
We study electron tunneling through a planar magnetic and electric barrier on the surface of a threedimensional topological insulator. For the double barrier structures, we find (i) a directional-dependent tunneling which is sensitive to the magnetic field configuration and the electric gate voltage, (ii) a spin rotation controlled by the magnetic field and the gate voltage, (iii) many Fabry-Pérot resonances in the transmission determined by the distance between the two barriers, and (iv) the electrostatic potential can enhance the difference in the transmission between the two magnetization configurations, and consequently lead to a giant magnetoresistance. Points (i), (iii), and (iv) are alike with that in graphene stemming from the same lineardispersion relations.
\end{abstract}

DOI: 10.1103/PhysRevB.82.115211

PACS number(s): 72.25.Dc, 73.20.At, 73.23.Ad, 75.47.De

\section{INTRODUCTION}

The topological insulator, a recent discovery of a new quantum state of matter, has attracted a growing interest due to its great scientific importance. ${ }^{1-7}$ In a topological insulator, spin-orbit coupling reverse the conduction and valence bands and opens a gap in the bulk. Surprisingly, the helical surface states appear in the bulk gap in the absence of magnetic fields, and are topologically protected by time-reversal symmetry, ${ }^{6-9}$ and are distinct from conventional surface states, which are fragile and depend sensitively on the details of the surface geometry and bonding. Topological insulators are interesting from the viewpoint of fundamental physics as well as potential applications in electronic devices.

The surface states in Bi-based alloys, $\mathrm{Bi}_{1-x} \mathrm{Sb}_{x}, \mathrm{Bi}_{2} \mathrm{Se}_{3}$, and $\mathrm{Bi}_{2} \mathrm{Te}_{3}$, were studied theoretically ${ }^{9,10}$ and experimentally by using angle-resolved photoemission spectroscopy. ${ }^{11-14}$ The surface states of the three-dimensional (3D) topological insulators (TIs) exhibit Dirac cone-shaped conduction and valence bands that meet at the $\Gamma$ point. Note that this Hamiltonian appears to be similar as the one for graphene, ${ }^{16,15}$ but topological insulators have an odd number of massless Dirac cones on the surface, ensured by the $Z_{2}$ topological invariant of the bulk, while graphene has twofold massless Dirac cones at the $K$ and $K^{\prime}$ valleys. Another essential difference is the status of the electron spin. In the surface Hamiltonian of the 3D TI, $\boldsymbol{\sigma}$ acts on the real spin of the charge carriers while for graphene it stands for the pseudospin, i.e., the A and B sublattices of graphene. Hence, topological insulators are expected to show some spin-relevant properties due to the spinmomentum locking, which is a consequence of their spindependent electron structure. ${ }^{6-8,17,18}$ These spin-dependent properties are absent in single-layer graphene. ${ }^{19-24}$

In this work, we study electron tunneling through two types of magnetic and electric double barriers where we considered square-shaped and delta-function-shaped magnetic fields. The square-shaped magnetic fields can be created by depositing a superconducting plate interrupted by two strips above the 3D TI in the presence of a magnetic field with magnetization perpendicular to the surface, neglecting the shrinking effect at the edges. Note that the magnetic field is restricted by the superconductor critical magnetic field $H_{c 1}$. However, from recent experiments, one can find that some materials can sustain superconductivity at very high magnetic field, for instance, $\mathrm{Nb}_{3} \mathrm{Sn}$ of which the $H_{c 1}$ could be up to $5.7 \mathrm{~T} .{ }^{25}$ The delta-function-shaped magnetic field is a simplified model for the magnetic field profile created by depositing two ferromagnetic metallic strips on the surface of a 3D TI with the magnetization parallel to the surface. The ferromagnetic strips are electrically isolated from the topological insulator through, e.g., a thin oxide layer. We investigate theoretically the transmission and conductance for parallel and antiparallel magnetic field configurations. We find that the transmission of electrons through the double barrier structures depends sensitively on the incident angle, the Fermi energy, the magnetic fields, and the electric gate voltages. The tunneling processes exhibit momentum and spin filtering behavior caused by the inhomogeneous magnetic field and the spin-momentum locking determined by the Hamiltonian of the 3D TI surface state. The tunneling magnetoresistance in such systems can be tuned significantly by changing the magnetic field and the height of the electric potential.

The paper is organized as follows. In Sec. II we present the theory of electron tunneling through magnetic and electric double barrier structures. In Sec. III, we show the numerical results and present our discussions. Finally, we give a brief conclusion in Sec. IV.

\section{THEORY}

We consider a double magnetic barrier with squareshaped or delta-function-shaped magnetic field on the surface of a 3D TI, as shown in Fig. 1. Such magnetic field profiles approximate those realized by depositing a patterned superconducting plate above the 3D TI in the presence of a perpendicular magnetic field or by depositing two ferromagnetic metal strips with the magnetization parallel to the surface on top of a 3D TI. Thus, we can create magnetic potentials underneath the superconducting plate or the ferromagnetic strips. ${ }^{26,27}$ In all cases, the created relevant magnetic fields are directed perpendicular to the surface of 
(a)

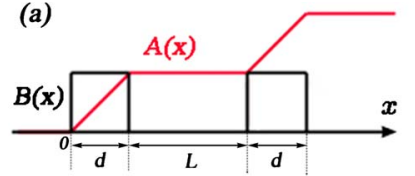

(b)

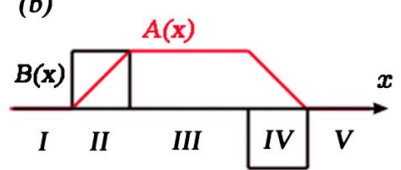

(e) (c)

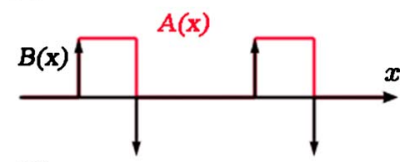

(d)

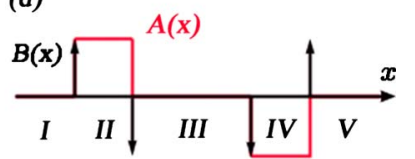

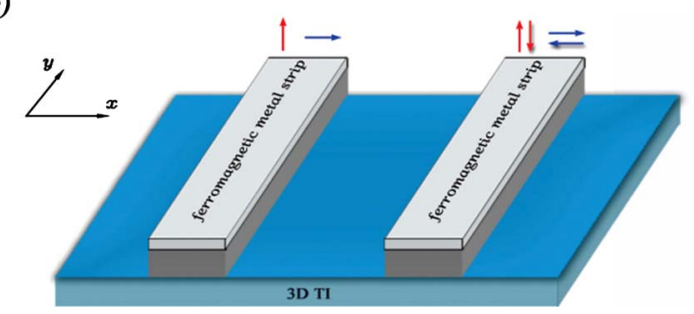

FIG. 1. (Color online) (e) Schematic illustration of the considered double barrier structure created by two ferromagnetic metallic strips on the surface of a 3D TI. The red vertical/blue horizontal arrows denote the magnetization directions of the strips that generate a double square-shaped/delta-function-shaped magnetic barrier. (a) Parallel configuration for the square-shaped barriers. (b) The same as in (a) but for antiparallel configuration. (c) Parallel configuration for the delta-function-shaped barriers. (d) The same as in (c) but for the antiparallel configuration.

the 3D TI. The low-energy electrons near the $\Gamma$ point of the Dirac cones can be well described by the effective Hamiltonian, ${ }^{6-8,28}$

$$
H=v_{F} \sigma \cdot(\boldsymbol{\pi} \times \hat{z})+V+H_{Z},
$$

where $v_{F}$ is the Fermi velocity, $\sigma^{i}(i=x, y, z)$ are the Pauli matrices denoting the real spin of the electrons, $V$ is the gate voltage applied on the magnetic metal strips, and the last term $H_{Z} \equiv g \mu_{B} \sigma \cdot \boldsymbol{B}$ is induced by Zeeman spin spitting. Note that for $g=23$ in $\mathrm{Bi}_{2} \mathrm{Se}_{3}$, the Zeeman term affect the transmission slightly at low magnetic field. The momentum is $\boldsymbol{\pi}=\mathbf{p}+\mathbf{e} \boldsymbol{A}$, where the vector potential $\boldsymbol{A}=\left(0, A_{y}, 0\right)$ is generated by the magnetic metal strips.

For simplicity, we introduce dimensionless units: $l_{B}$ $=\left[\hbar / e B_{0}\right]^{1 / 2}, B(x) \rightarrow B_{0} B(x), A_{y}(x) \rightarrow B_{0} l_{B} A_{y}(x), E \rightarrow E_{0} E, \boldsymbol{r}$ $\rightarrow l_{B} \boldsymbol{r}, \boldsymbol{k} \rightarrow \boldsymbol{k} / l_{B}$, so that the Hamiltonian becomes

$$
H=\left(\begin{array}{cc}
V+g \mu_{B} B & k_{y}+A_{y}+i k_{x} \\
k_{y}+A_{y}-i k_{x} & V-g \mu_{B} B
\end{array}\right) .
$$

In the presence of an inhomogeneous magnetic field, the components of the wave vector satisfy the relationship $k_{x}^{2}$ $+\left(k_{y}+A_{y}\right)^{2}=(E-V)^{2}-\left(g \mu_{B} B\right)^{2}$. Note that the translational invariance along the $y$ direction gives rise to conservation of the momentum $k_{y}$ along the interface.

In this work we consider two extreme cases for the magnetic field profile: (1) square-shaped magnetic barrier and (2) delta-function magnetic barrier profiles. First we consider electron transmission through a double constant-field magnetic barrier, i.e., $V=0$ and $B_{z}(x)=B\{[\theta(x-d)-\theta(x)] / 2$ $+\gamma[\theta(x-2 d-L)-\theta(x-d-L)] / 2\}$, which are schematically

shown in Figs. 1(a) and 1(b), where $\gamma= \pm 1$ represents the magnetization configuration corresponding to the parallel and/or antiparallel configurations. In the free region I [see Fig. 1(a)], the vector potential $A_{y}$ is a constant, the corresponding energy is $E_{ \pm}= \pm v_{F} \pi$, and the wave function is,

$$
\psi_{\mathrm{I}}=\frac{1}{\sqrt{2}}\left(\begin{array}{c}
1 \\
\frac{-i k_{x}+\left(k_{y}+A_{y}\right)}{E_{F}-V}
\end{array}\right) e^{i k_{x} x}+\frac{r}{\sqrt{2}}\left(\begin{array}{c}
1 \\
\frac{i k_{x}+\left(k_{y}+A_{y}\right)}{E_{F}-V}
\end{array}\right) e^{-i k_{x} x} .
$$

In the barrier region II, the vector potential is given by $\boldsymbol{A}$ $=(0, B x, 0)$ and the wave function can be expressed in terms of the parabolic cylinder functions $D_{\nu}$,

$$
\psi_{\mathrm{II}}=\sum_{ \pm} c_{ \pm}\left(\begin{array}{c}
D_{v / 2-1}\left[ \pm \sqrt{2}\left(x+k_{y}\right)\right] \\
\pm \sqrt{\frac{2}{v}} D_{v / 2}\left[ \pm \sqrt{2}\left(x+k_{y}\right)\right]
\end{array}\right)
$$

with the complex coefficients $c_{ \pm}$and $v \equiv\left(E_{F}-V\right)^{2}$ $-\left(g \mu_{B} B\right)^{2}$. In regions III, IV, and V, the corresponding wave functions can be obtained by repeating a similar procedure. The scattering-matrix technique is used to calculate the transmission probability. ${ }^{29}$

In order to understand the effect of the magnetic field configurations on the electron tunneling, we also considered a double magnetic barrier with delta-function-shaped magnetic fields at the edges of the barriers as shown in Figs. 1(c) and 1(d). The magnetic field is perpendicular to the surface and is given by $B_{z}(x)=B\{[\delta(x)-\delta(x-d)] / 2+\gamma[\delta(x-d-L)$ $-\delta(x-2 d-L)] / 2\}$. Note that the vector potential is a constant in the barrier regions, in contrast to the linear function in the barrier for magnetic barriers with constant field. The electron wave function in the different regions is similar to that of the square-shaped magnetic barriers obtained above [see Eqs. (3) and (4)].

The ballistic conductance at finite temperature is calculated from the Landauer-Büttiker formalism, ${ }^{30}$

$$
G\left(E_{F}\right)=G_{0} \int_{-\infty}^{\infty} \int_{-k_{F}}^{k_{F}} T\left(E_{F}, k_{y}\right) F_{T}\left(E-E_{F}\right) d E d k_{y},
$$

where $G_{0} \equiv \frac{e^{2} L_{y}}{\pi h}$ is taken as the conductance unit, $L_{y}$ is the sample size along the $y$ direction which is much larger than $L$ and $d, F_{T}\left(E-E_{F}\right)=-d f(E) / d E$ is the thermal broadening function, and $f(E)$ is the Fermi-Dirac distribution function.

\section{RESULTS AND DISCUSSIONS}

First we consider the tunneling process through a double square-shaped magnetic barrier with parallel configuration. The transmission probabilities are shown in Figs. 2(a) and 2(b) for a magnetic barrier $(B=1)$ with the width $d=1$ and the distances between the two barriers $L=3 d$ and $9 d$, respectively. We find that the transmission becomes asymmetric with respect to the in-plane momentum $k_{y}$ along the interface since the magnetic field leads to a momentum-filtering feature. When the vector potential $A_{y}$ exceeds a critical value, the outgoing state becomes an evanescent wave decaying 
(a)

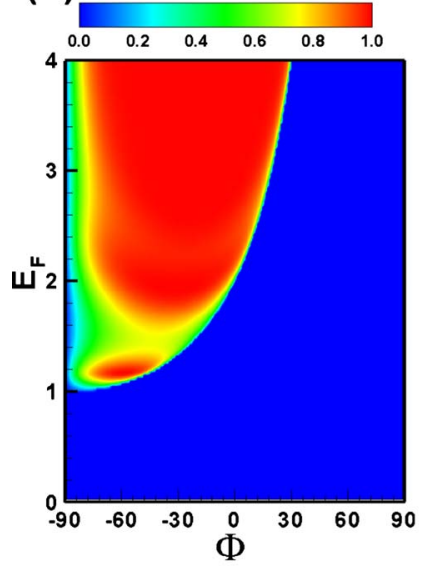

(b)

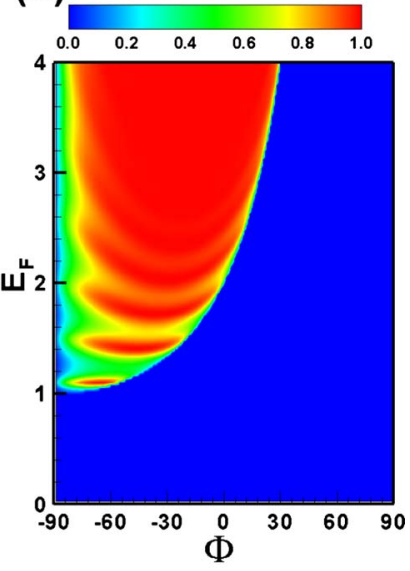

(c)

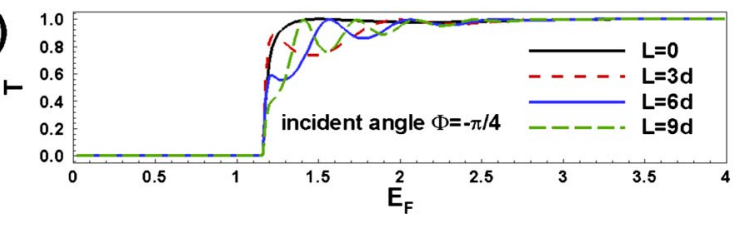

FIG. 2. (Color online) (a) The contour plot of the transmission probability through a parallel square-shaped magnetic double barrier as a function of the incident angle and incident energy, for a fixed barrier width $d=1$, distance $L=3$, and magnetic field $B=1$. The magnetic unit $B_{0}=1 \mathrm{~T}$, the energy unit is $E_{0}=16 \mathrm{meV}$, and the length unit is $l_{B}=26 \mathrm{~nm}$. (b) The same as panel (a) but for the distance $L=9$. (c) The transmission as a function of the incident energy $E_{F}$ for a fixed incident angle $\Phi=-\pi / 4$ for different distances between the two magnetic barriers.

spatially along the propagating direction [see Fig. 2(a)]. It is interesting to notice that tunneling is forbidden for a certain incident angle $\Phi$ and incident energy $E_{F}$, i.e., a wave-vector filtering behavior is found. The boundary of the total reflection region $(T=0)$ can be approximately given by the relationship $k_{y}+B d+B d=E_{F}$. The inhomogeneous magnetic field results in an asymmetric behavior of the transmission as a function of the incident angle. In the tunneling-forbidden region, the transmitted electrons are in the evanescent modes which decay exponentially along the propagating direction. The total reflection can be understood by the semiclassical picture: the electron will move on a cyclotron orbit in the barrier region. If the cyclotron orbit radius $R_{c}<d$, the incident electron will exit the barrier region backward eventually. ${ }^{31}$ Note that this cyclotron motion will suppress the transmission regardless of the incidence angle $\Phi$. Interestingly, the transmission decreases and even vanishes as the magnetic field increases. This behavior implies that the Dirac fermions can be confined by the inhomogeneous magnetic field while electrons can transmit perfectly through the electric barrier because of Klein tunneling. The transmission for larger distance $L$ between the two barriers [see Fig. 2(b)] exhibits more significant oscillation compared to that of the small $L$ case [see Fig. 2(a)] for the same incident angle. This is caused by the Fabry-Pérot resonant modes formed between the two barriers due to the multiple reflections between the two interfaces. In Fig. 2(c), we show the FabryPérot resonances for different distances at a fixed incident (a)

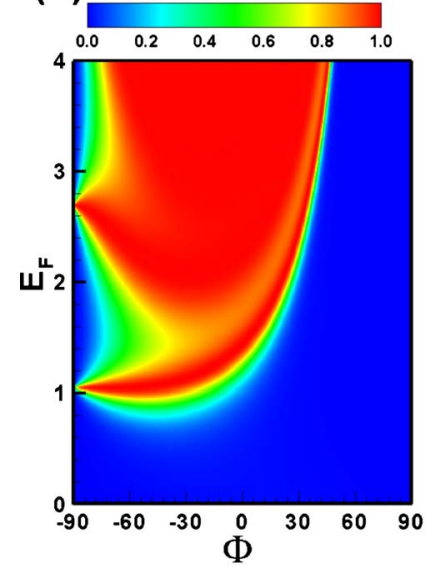

(b)

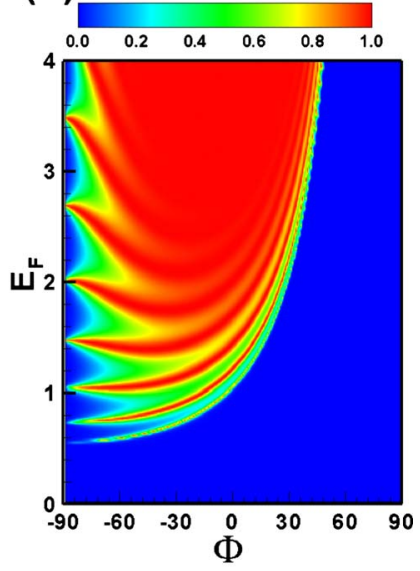

(c)

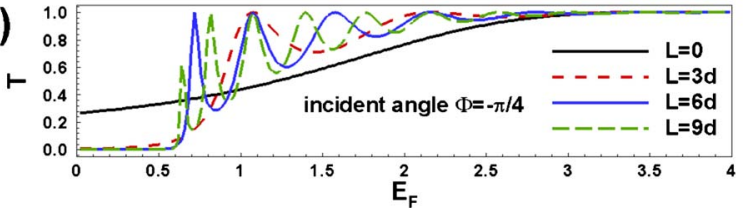

FIG. 3. (Color online) The same as Fig. 2 but for the antiparallel configuration.

angle $\Phi=-\pi / 4$. For a single barrier structure (i.e., $L=0$ ), there is no oscillating behavior, because no quasibound states exist between the two barriers. If $L \neq 0$, Fabry-Pérot modes appear and become more significant as the distance $L$ increases.

Next we discuss the transmission through double squareshaped magnetic barriers with antiparallel configuration. The transmission [see Figs. 3(a) and 3(b)] becomes very different from that of the parallel configuration. The parallel and antiparallel configurations strongly affect the perfect transmission region, and therefore provides us with a possible way to control the transmission by simply reversing the magnetization configuration of the ferromagnetic strips. The vector potential is depicted in Fig. 1(b). As the vector potentials are the same on each side of the double barrier, i.e., the antiparallel configuration, the outgoing wave is always in the propagating modes. Electrons can tunnel through the double barrier structure with the antiparallel configuration even when the electron wave vector $k_{x}$ is imaginary, i.e., evanescent modes, in the middle region between the two barriers. Therefore, electrons can more easily tunnel through the double barriers in case of an antiparallel configuration than that for the parallel configuration. The boundary of the tunnelingforbidden region can be approximately given by the relationship $k_{y}+B d=E_{F}$. Figure 3(c) shows the transmission as a function of the incident energy at different distances between two barriers. Notice that there are more pronounced resonant peaks caused by the Fabry-Pérot modes formed between the two barriers with increasing distance $L$ and larger peak-tovalley ratio than that for the parallel configuration case.

Note that the regions of the transmission gaps are completely different between the two magnetization configurations. This is because the total reflection for the parallel configuration stems from the evanescent modes in the outgoing region (the region $\mathrm{V}$ ) while that for the antiparallel configu- 
(a)

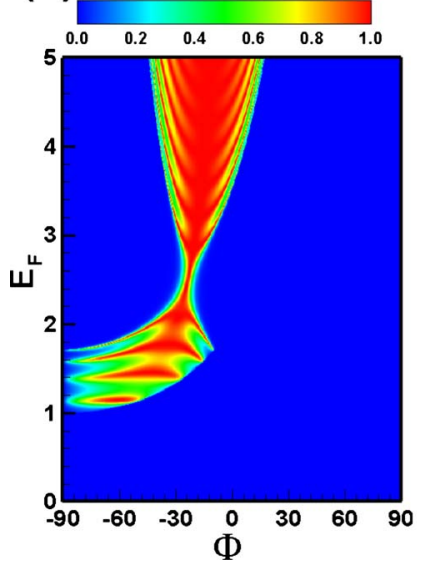

(b)

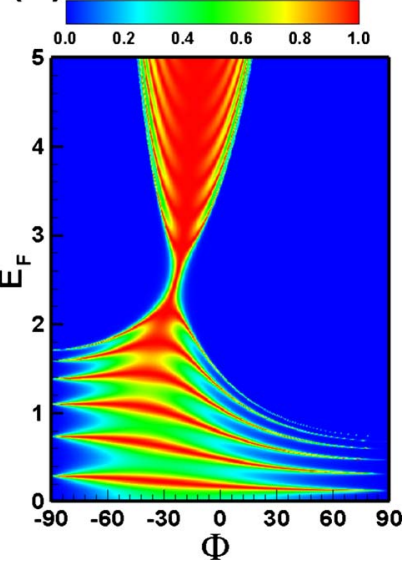

FIG. 4. (Color online) (a) The same as Fig. 2(b), i.e., the parallel configuration but in the presence of a potential barrier $V_{3}=2.5$. (b) The same as Fig. 3(b), i.e., the antiparallel configuration but now with $V_{3}=2.5$.

ration is determined by the evanescent modes in the middle region (the region III).

It is interesting to see the effect of the electrostatic potential on the perfect transmission and total reflection. In Fig. 4, we plot the transmission probability as a function of the incident angle $\Phi$ and incident energy $E_{F}$ in case of a nonzero electrostatic potential $V_{3}=2.5$ applied in region III. The transmission is heavily suppressed in an energy interval around the electrostatic potential for the two magnetization configurations. According to the relationship $k_{x}^{2}+\left(k_{y}+A_{y}\right)^{2}$ $=(E-V)^{2}$, the wave vector $k_{x}$ tends to be imaginary with a larger absolute magnitude as the incident energy becomes closer to the electrostatic potential. Therefore, the evanescent modes decay more rapidly in the middle region and lead to the suppression of transmission [see Figs. 2(b), 3(b), and 4]. In particular, at low incident energy, the transmission behaviors are significantly different for the two magnetization configurations. When the electrostatic potential $V_{3}$ is large enough, the evanescent modes in region III are turned into the transmission modes. We find that there are many transmission peaks at the low-energy regime induced by the electrostatic potential for antiparallel configuration [see Fig. 4(b)] since the low-energy transmission-forbidden region arises from the evanescent modes in the middle region. Such behavior offers us a possible way to construct an electric switching device. Notice that the low-energy transmission gap is almost not modified, e.g., the boundary of the lowenergy tunneling-forbidden region, when the same electrostatic potential is applied [see Fig. 4(a)]. This is because the total reflection is caused by the evanescent modes in the outgoing region (region $\mathrm{V}$ ) that are almost unaffected by the electrostatic potential in region III. Thus, the electrostatic potential can enhance the difference in the transmission between the two magnetization configurations.

Interestingly, we can manipulate spin transport on the surface of a 3D topological insulator by controlling the electron orbital motion since $\boldsymbol{\sigma} \cdot \boldsymbol{k}$ is a conserved quantity, i.e., there is spin-momentum locking. ${ }^{17}$ Figures 5(a) and 5(b) show the spin orientation as function of the incident angles and ener-

gies for a double square-shaped magnetic barrier with parallel configuration for different electrostatic potentials $V_{3}=0$ and 3 , respectively. From the spin orientation one can see that the transmitted electron spin no longer points along the same direction of the incident electron spin, but is rotated over an angle, which is determined by the incident angle, the electron energy and the vector potential $A_{y}$. Note that $A_{y}$ in the region $\mathrm{V}$ is determined by the magnetic barrier height and width, so changing the size of the barrier and/or the magnetic field can tune the spin and direction of motion of the transmitted electrons. The electrostatic potential can strongly reduce the transmission probability for an incident energy that is close to the value of the electrostatic potential $V_{3}$. This feature provides us with a new way to realize directional filtering of electrons. Electrons with certain incident angles and incident energies can be transmitted to region $\mathrm{V}$ with a rotated spin orientation [see Fig. 5(b)]. The numerical results for the double square-shaped magnetic barrier with antiparallel configuration are shown in Figs. 5(c) and 5(d). We find that the transmitted electron spin are polarized along the same direction as the incident electron spin, indicating that the double magnetic barrier with antiparallel configuration is unable to rotate the electron spin, the reason being that the first and second magnetic barriers bend the trajectory of the electron in opposite direction, therefore the outgoing electron will propagate in the same direction as the incident electron, and the transmitted electron spin is unchanged due to the spin-momentum locking. In the presence of an electrostatic potential, the total reflection regions are significantly reshaped as shown in Fig. 5(d). Note that for the antiparallel configuration, the low-energy electrons can transmit through the double barrier with a rotated spin orientation, while for the parallel configuration, the low-energy transmission gap is almost unchanged when such an electrostatic potential is applied. The spin orientation of the reflected electron, with reversed longitudinal wave vector and conserved transverse wave vector, is always rotated due to the spin-momentum locking.

Next we will consider tunneling through the deltafunction-shaped magnetic barriers both for parallel and antiparallel configurations. For parallel configuration as sketched in Fig. 1(c), the transmission spectra display strong anisotropic behavior with respect to the incident angle [see Fig. $6(\mathrm{a})]$. In the barrier region, the $y$-component momentum is equal to $k_{y}+A_{y}$, so electrons with positive incident angles, i.e., $k_{y}>0$, are more likely to transmit through evanescent modes, corresponding to the condition $k_{y}+A_{y}>E_{F}$, in the barriers. The transmission exhibits obvious Fabry-Pérot resonant behavior which is very different from that for the square-shaped magnetic barrier case, where tunneling is totally forbidden for large positive incident angles or low incident energies (see Figs. 2 and 3). As the barrier length $d$ increases, the evanescent modes in the barriers reduce the transmission probability more significantly, and thus the resonant peaks in the region with large positive incident angles and low incident energies will disappear as shown in Fig. 6(b). In this case we find a similar boundary as outlined above. When we reverse the magnetization direction of the second ferromagnetic strip, the magnetization configuration is easily switched to the antiparallel configuration as shown 
(a)

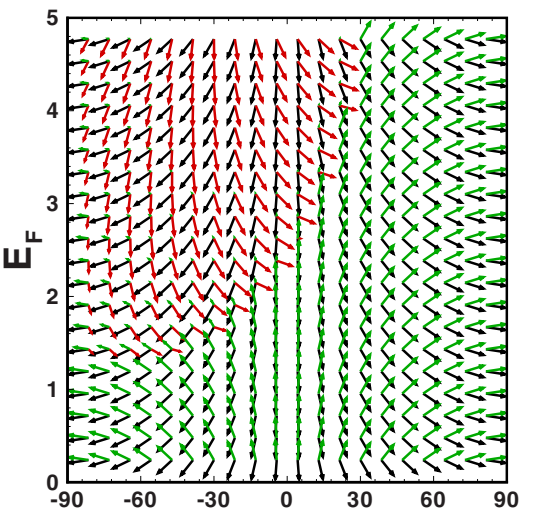

(c)

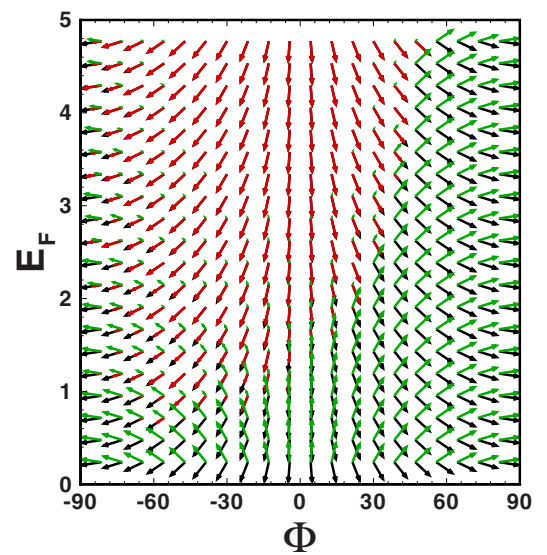

(b)

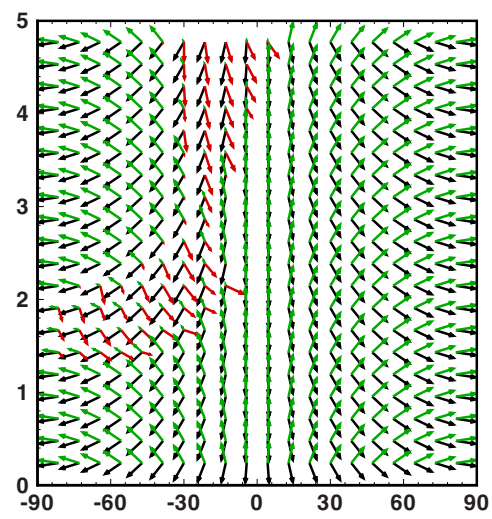

(d)

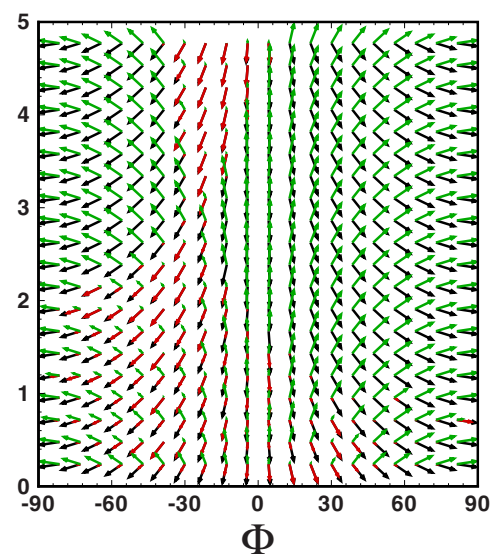

FIG. 5. (Color online) The spin orientations of the incident (black arrows), transmitted (red arrows), and the reflected (green arrows) electrons as a function of the incident angle and energy, for a fixed barrier width $d=1$, distance $L=9$, and magnetic field $B=1$. For the parallel configuration, (a) $V_{3}=0$, (b) $V_{3}=2.5$, and for the antiparallel configuration, (c) $V_{3}=0$, (d) $V_{3}=2.5$. in Fig. 1(d). For this configuration, the vector potential $A_{y}(x)$ is antisymmetric about the center of the whole structure. The Hamiltonian possesses a symmetry associated with the operation $\hat{T} \hat{R}_{x} \hat{\sigma}_{y}$, where $\hat{T}$ is the time-reversal operator, $\hat{R}_{x}$ is the reflection operator about the center between the two barriers, and $\hat{\sigma}_{y}$ is one of the Pauli matrices. This symmetry implies (a)

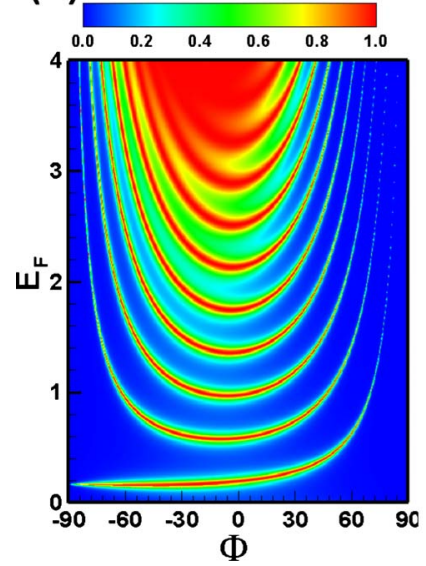

(b)

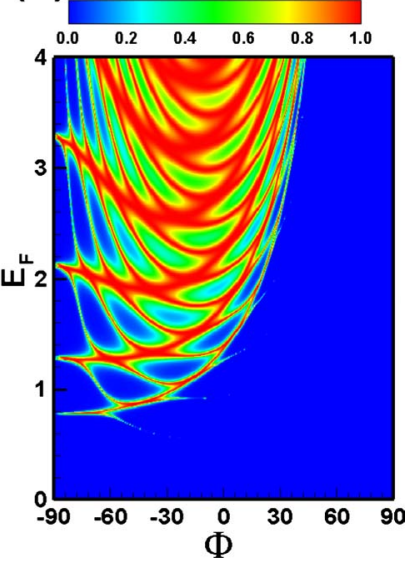

FIG. 6. (Color online) (a) The contour plot of the transmission through a parallel delta-function-shaped magnetic double barrier as a function of the incident angle and energy, for the barrier width $d=1$, the distance $L=9$, and the magnetic field $B=1$. (b) The same as panel (a) but with the barrier width $d=9$. the invariance of the transmission with respect to the replacement $k_{y} \rightarrow-k_{y}$. Our numerical results demonstrate this symmetric feature (see Fig. 7). We also found that the FabryPérot resonances become more pronounced as the distance between the two barriers increases.

Next we discuss the transmission through a combined electric and delta-function-shaped magnetic double barrier. The electrostatic potentials are applied in the same region as (a)

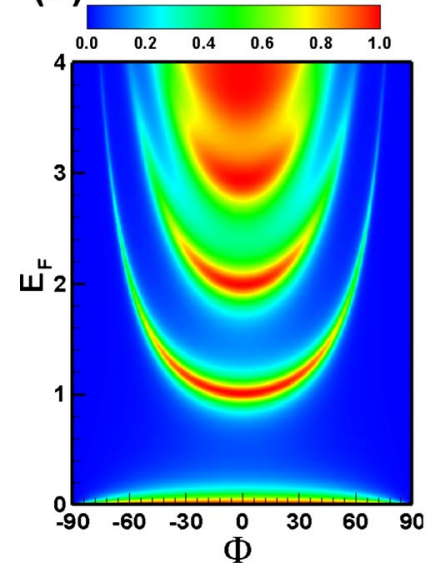

(b)

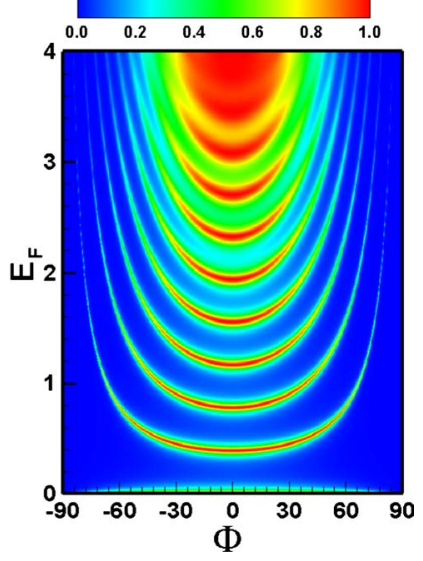

FIG. 7. (Color online) The same as Fig. 6 but for the antiparallel configuration. The barrier width $d=1$ and the distances are: (a) $L$ $=3$ and (b) $L=9$, respectively. 
(a)

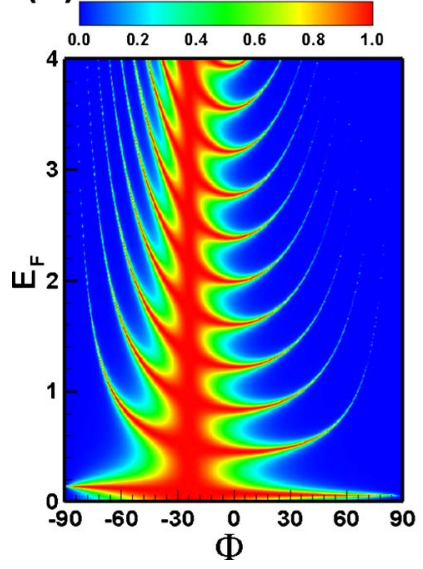

(b)

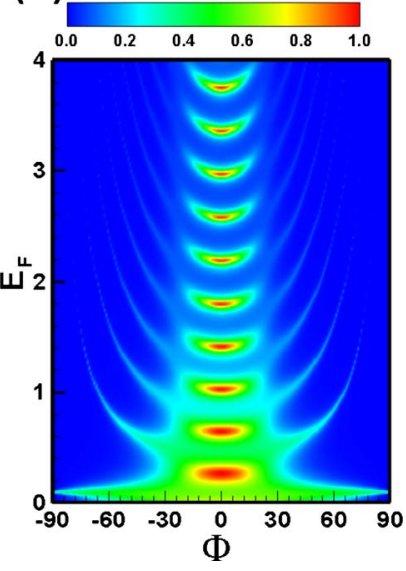

FIG. 8. (Color online) (a) The same as Fig. 6(a), i.e., the parallel configuration but for $V_{2}=V_{4}=3$. (b) The same as Fig. 7(b), i.e., the antiparallel configuration but for $V_{2}=V_{4}=3$.

the magnetic double barrier. In the barrier region, the longitudinal wave vector is given by $k_{x}$ $=\sqrt{\left(E_{F}-V\right)^{2}-\left(E_{F} \cdot \sin \Phi+A_{y}\right)^{2}}$ with $A_{y}<V$ in dimensionless units. The vector potentials are the same in the two barriers for parallel configuration. We notice that for certain incident angle $\Phi=\arcsin \left(-A_{y} / V\right)$, the longitudinal wave vector $k_{x}$ $=\sqrt{1-\left(A_{y} / V\right)^{2}} \cdot\left|E_{F}-V\right|$, is always a real number. Electrons can always transmit via propagating states in the barrier region around this incident angle regardless of the incident energy. Our numerical results agree with this analytical derivation [see Fig. 8(a)]. But for antiparallel alignment, there is no such angle-selected transmission channel, since the vector potentials are opposite in the two barriers [see Fig. 8(b)].

Interestingly, one can see that the transmission decreases as the incident energy increases. This is because evanescent modes appear when the incident energy approaches the height of the electric barrier $\left(V_{2}=V_{4}=3\right)$, which suppresses the transmission in such incident energy interval.

Finally, we focus on the conductance $G$ and the magnetoresistance $\mathrm{MR}=\left(G_{P}-G_{A P}\right) / G_{A P}$, where the subscript $P$ $(A P)$ denotes parallel (antiparallel) configuration. In Fig. 9, the conductance $G_{P}, G_{A P}$ and magnetoresistance ratio MR are plotted as a function of the Fermi energy for different heights of the electric and magnetic barriers. For a squareshaped double barrier, the conductance for both parallel and antiparallel configurations exhibit slightly oscillating behaviors and the magnetoresistance ratio MR is small over the calculated energy region as shown in Figs. 9(a) and 9(b). This feature is caused by the structure in the transmission discussed above. The zero conductance at low Fermi energy corresponds to the transmission gap shown in Figs. 2 and 3. For a delta-function-shaped double barrier, the conductances exhibit significant oscillations for both parallel and antiparallel configurations [see the blue dashed curves in Figs. 9(c)-9(f)]. Note that the position of the conductance peaks and valleys are distinct for the two magnetic configurations. This feature comes from the difference in transmission between the parallel and antiparallel configurations in the different energy regions. The magnetoresistance shows many peaks which are found for the Fermi energies corresponding to the peaks (valleys) of $G_{P}\left(G_{A P}\right)$ [see red solid curves in Figs. 9(c)-9(f)]. We also find that a strong magnetic field can effectively increase the magnetoresistance MR. Since a large magnetic field results in large imaginary wave vectors for the evanescent modes in the barrier region and strongly suppress the transmission probability. Therefore, the conductance $G_{A P}$
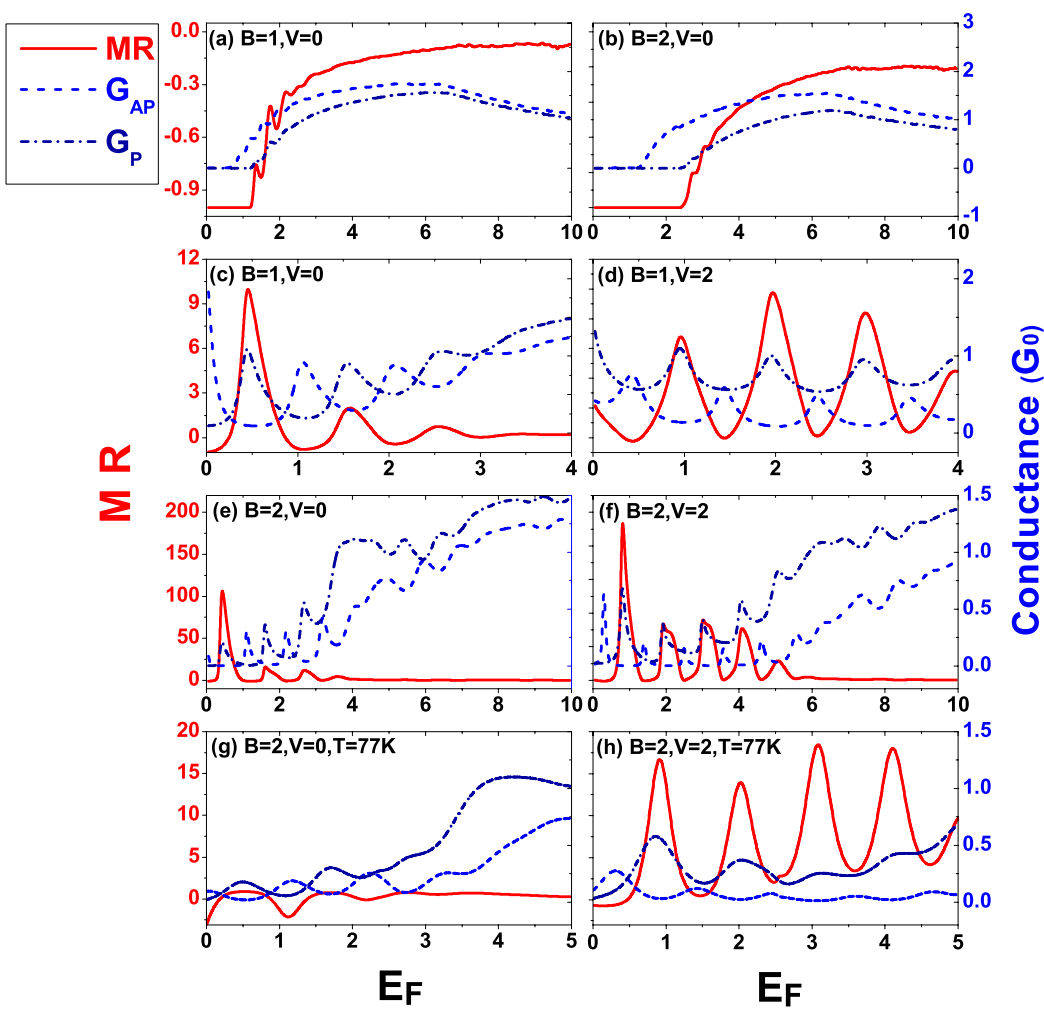

FIG. 9. (Color online) (a) The tunneling conductance $G_{P}, G_{A P}$ (the blue dashed curves) and magnetoresistance ratio MR (the red solid curve) as a function of the incident energy $E_{F}$ in the presence of a double square-shaped magnetic/ electric barrier, for $d=1, L=9, B=1$, and $V=0$. Here, we set $V_{1}=V_{3}=V_{5}=0$ and $V_{2}=V_{4}=V$. (b) The same as panel (a) but for $B=2$. [(c)-(h)] The dependence on the incident energy $E_{F}$ of the conductance and MR in the presence of a double delta-function-shaped barrier, for $d=1, L=3$, (c) $B=1, V=0$; (d) $B=1, V=2$; (e) $B=2, V=0$; (f) $B=2, V=2$; (g) and (h) the same as the panels (e) and (f) but for a finite temperature $T=77 \mathrm{~K}$. The magnetic unit $B_{0}=0.5 \mathrm{~T}$, the energy unit is $E_{0}$ $=11 \mathrm{meV}$, and the length unit is $l_{B}=36 \mathrm{~nm}$. 
at the valleys are close to zero and thus significantly increase MR. A giant magnetoresistance MR 200 can be achieved under such configuration. The electrostatic potential for the same magnetic barrier can shift the transmission gaps, enhance the difference of the transmission between parallel and antiparallel configurations, and thus can be used to adjust the MR efficiently. Finally, we estimate the effect of temperature on the conductance of the delta-function-shaped double magnetic barrier that shows a huge magnetoresistance MR at zero temperature. Our numerical results show that thermal fluctuations broaden the conductance peaks, making the magnetoresistance MR drop about one order of magnitude at $T=77 \mathrm{~K}$ [see Figs. $9(\mathrm{~g})$ and $9(\mathrm{~h})]$.

\section{CONCLUSION}

In summary, we investigated theoretically the quantum tunneling processes through two types of double magnetic/ electric barriers on the surface of a three-dimensional topological insulator. For both square- and delta-function-shaped magnetic barriers, the transmission shows (i) a directionaldependent tunneling depending sensitively on the magnetic field configurations and the electric gate voltage, (ii) a spin rotation locked with electron momentum, (iii) many FabryPérot resonances in the transmission determined by the distance between the two barriers, and (iv) the electrostatic potential can enhance the difference of the transmission between the two magnetization configurations, and consequently lead to a giant magnetoresistance, which provides us with a new way to construct a topological insulator-based wave-vector filter and magnetoresistance device.

\section{ACKNOWLEDGMENTS}

This work was supported by the NSF of China, the Flemish Science Foundation (FWO-Vl), and the Belgian Science Policy.
${ }^{1}$ X. L. Qi and S. C. Zhang, Phys. Today 63(1), 33 (2010); M. Hasan and C. Kane, arXiv:1002.3895 (unpublished).

${ }^{2}$ C. L. Kane and E. J. Mele, Phys. Rev. Lett. 95, 146802 (2005).

${ }^{3}$ C. L. Kane and E. J. Mele, Phys. Rev. Lett. 95, 226801 (2005).

${ }^{4}$ B. A. Bernevig and S. C. Zhang, Phys. Rev. Lett. 96, 106802 (2006).

${ }^{5}$ B. A. Bernevig, T. Hughes, and S. C. Zhang, Science 314, 1757 (2006).

${ }^{6}$ J. E. Moore and L. Balents, Phys. Rev. B 75, 121306(R) (2007).

${ }^{7}$ L. Fu, C. L. Kane, and E. J. Mele, Phys. Rev. Lett. 98, 106803 (2007).

${ }^{8}$ X. L. Qi, T. L. Hughes, and S. C. Zhang, Phys. Rev. B 78, 195424 (2008).

${ }^{9}$ L. Fu and C. L. Kane, Phys. Rev. B 76, 045302 (2007),

${ }^{10}$ H. J. Zhang, C. X. Liu, X. L. Qi, X. Dai, Z. Fang, and S. C. Zhang, Nat. Phys. 5, 438 (2009).

${ }^{11}$ D. Hsieh, D. Qian, L. Wray, Y. Xia, Y. S. Hor, R. J. Cava, and M. Z. Hasan, Nature (London) 452, 970 (2008).

${ }^{12}$ D. Hsieh, Y. Xia, L. Wray, D. Qian, A. Pal, J. H. Dil, J. Osterwalder, F. Meier, G. Bihlmayer, C. L. Kane, Y. S. Hor, R. J. Cava, and M. Z. Hasan, Science 323, 919 (2009).

${ }^{13}$ Y. Xia, D. Qian, D. Hsieh, L. Wray, A. Pal, H. Lin, A. Bansil, D. Grauer, Y. S. Hor, R. J. Cava, and M. Z. Hasan, Nat. Phys. 5, 398 (2009).

${ }^{14}$ Y. L. Chen, J. G. Analytis, J. H. Chu, Z. K. Liu, S. K. Mo, X. L. Qi, H. J. Zhang, D. H. Lu, X. Dai, Z. Fang, S. C. Zhang, I. R. Fisher, Z. Hussain, and Z. X. Shen, Science 325, 178 (2009).

${ }^{15}$ C. W. Beenakker, Rev. Mod. Phys. 80, 1337 (2008).

${ }^{16}$ A. H. Castro Neto, F. Guinea, N. M. R. Peres, K. S. Novoselov, and A. K Geim, Rev. Mod. Phys. 81, 109 (2009).

${ }^{17}$ D. Hsieh, Y. Xia, D. Qian, L. Wray, J. H. Dil, F. Meier, J.
Osterwalder, L. Patthey, J. G. Checkelsky, N. P. Ong, A. V. Fedorov, H. Lin, A. Bansil, D. Grauer, Y. S. Hor, R. J. Cava, and M. Z. Hasan, Nature (London) 460, 1101 (2009).

${ }^{18}$ S. Mondal, D. Sen, K. Sengupta, and R. Shankar, Phys. Rev. Lett. 104, 046403 (2010); arXiv:1003.2277 (unpublished).

${ }^{19}$ F. Zhai and K. Chang, Phys. Rev. B 77, 113409 (2008).

${ }^{20}$ M. Ramezani Masir, P. Vasilopoulos, and F. M. Peeters, Appl. Phys. Lett. 93, 242103 (2008).

${ }^{21}$ M. Ramezani Masir, P. Vasilopoulos, and F. M. Peeters, New J. Phys. 11, 095009 (2009).

${ }^{22}$ S. Ghosh and M. Sharma, J. Phys.: Condens. Matter 21, 292204 (2009).

${ }^{23}$ L. Dell'Anna and A. De Martino, Phys. Rev. B 79, 045420 (2009).

${ }^{24}$ L. Dell'Anna and A. De Martino, Phys. Rev. B 80, 155416 (2009).

${ }^{25}$ A. Godeke, Supercond. Sci. Technol. 19, R68 (2006).

${ }^{26}$ P. D. Ye, D. Weiss, R. R. Gerhardts, M. Seeger, K. von Klitzing, K. Eberl, and H. Nickel, Phys. Rev. Lett. 74, 3013 (1995); A. Nogaret, J. Phys.: Condens. Matter 22, 253201 (2010).

${ }^{27}$ A. Matulis, F. M. Peeters, and P. Vasilopoulos, Phys. Rev. Lett. 72, 1518 (1994); J. Hong, S. Joo, T. S. Kim, K. Rhie, K. H. Kim, S. U. Kim, B. C. Lee, and K. H. Shinc, Appl. Phys. Lett. 90, 023510 (2007); M. Cerchez, S. Hugger, T. Heinzel, and N. Schulz, Phys. Rev. B 75, 035341 (2007), and references therein.

${ }^{28}$ I. Garate and M. Franz, Phys. Rev. Lett. 104, 146802 (2010).

${ }^{29}$ Lebo Zhang, P. Brusheim, and H. Q. Xu, Phys. Rev. B 72, 045347 (2005).

${ }^{30}$ M. Büttiker, Y. Imry, R. Landauer, and S. Pinhas, Phys. Rev. B 31, 6207 (1985).

${ }^{31}$ F. M. Peeters and A. Matulis, Phys. Rev. B 48, 15166 (1993). 